\title{
F12-related hereditary angioedema with normal C1Inh
}

INSERM

\section{Source}

INSERM. (1999). Orphanet: an online rare disease and orphan drug data base. F12related hereditary angioedema with normal C1Inh. ORPHA:100054

Hereditary ang ioedema type 3 (HAE 3) is a form of hereditary angioedema (see this term) characterized by acute edema in subcutaneous tissues, viscera and/or the upper airway. 